\title{
Levels of Conscience and Related Factors among Iranian Oncology Nurses
}

\author{
Behrang Gorbanzadeh ${ }^{1}$, Azad Rahmani²*, Sima Mogadassian¹, Mojhgan \\ Behshid $^{1}$, Arman Azadi ${ }^{3}$, Saied Taghavy ${ }^{4}$
}

\begin{abstract}
Background: Having a conscience is one of the main pre-requisite of providing nursing care. The knowledge regarding levels of conscience among nurses in eastern countries is limited. So, the purpose of this study was to examine the level of conscience and its related factors among Iranian oncology nurses. Materials and Methods: This descriptive-correlational study was conducted in 3 hospitals in Tabriz, Iran. Overall, 68 nurses were selected using a non-probability sampling method. The perceptions of conscience questionnaire was used to identify the levels of conscience among nurses. The data were analyzed using SPSS version 13.0. Results: The mean nurses' level of conscience scores was 72.7. In the authority and asset sub-scales nurses acquired higher scores. The mean of nurses' scores in burden and depending on culture sub-scales were the least. Also, there were no statistical relationship between some demographic characteristics of participants and their total score on the perceptions of conscience questionnaire. Conclusions: According to study findings Iranian nurses had high levels of conscience. However, understanding all the factors that affect nurses' perception of conscience requires further studies.
\end{abstract}

Keywords: Conscience - nurses - oncology - nursing ethics - Iran

Asian Pac J Cancer Prev, 16 (18), 8211-8214

\section{Introduction}

Nurses are one of the main components of healthcare system and a large part of the demands of patient care is centered on the work of nurses (Weaver, 2007; Ahlin et al., 2013). Nowadays, the delivery of high-quality care is expected (Jensen and Lidell, 2009). However, due to financial crisis and hospital Staff Downsizing in the recent decades, it has been expected that the vast majority of services be provided by nurses' population. Nurses today, more than ever, are facing challenging situations which requires difficult ethical decisions regarding patients care. One of the factors affecting the nursing responses to these ethically difficult situations is conscience (Juthberg et al., 2007). It is so important for the nurse to discuss about the meaning of conscience. Also nurses are considered the conscience of the health care system (Vanaki and Memarian, 2009).

Conscience is a concept in the field of medical ethics (Glasberg et al., 2006). A good conscience is associated with feelings of integrity and ethical responsibility and troubled conscience causes shame and guilt (Dahlqvist et al., 2009). Stress of conscience which refers to stress that is caused by a troubled conscience may arise from personal, social and organizational pressures (Tuvesson et al., 2012). Stress of conscience occurs when people are prevented from doing what they think is the right thing (Glasberg et al., 2006). Conscience has been described as an inner voice that must be interpreted and needs peace to be heard and cannot be avoided (Juthberg et al., 2008). Conscience is the inner voice which warns us about other people (Juthberg et al., 2007).

It should be noted that conscience is the backbone of medical ethics and can significantly affect nurses' personal and professional lives (Dahlqvist et al., 2009). Conscience is a prerequisite to ethical behavior and has an important role in providing accurate and secure nursing care (Dahlqvist et al., 2009). According to literature conscience is an asset that guided nurses in their efforts to provide high quality care (Jensen and Lidell, 2009). On the other hand, Suppression of conscience may cause nurses job frustration and thoughts of quitting (Juthberg et al., 2008). The finding of study revealed that Conscience can explain 42 percent of the variance in occupational belonging (Dahlqvist et al., 2009).

Like other nurses, nurses working in oncology departments should also have a high level of consciousness (Wengstrom and Ekedahl, 2006). Cancer patients have

${ }^{1}$ Medical-Surgical Department, Nursing and Midwifery Faculty, ${ }^{2}$ Hematology and Oncology Research Center, Tabriz University of Medical Sciences, ${ }^{4}$ Department of Psychology, Islamic Azad University, Tabriz branch, Tabriz, ${ }^{3}$ Department of Nursing, Ilam University of Medical Sciences, Ilam, Iran*For correspondence: azad.rahmani@yahoo.com 
many physical, psychological, social and spiritual needs and require high quality nursing care (Yeh et al., 1999; Abdollahzadeh et al., 2014; Abbasnezhad et al., 2015; Avestan et al., 2015; Fathollahzade et al., 2015). Cancer patients are very dependent on their caregivers and the low level of consciousness among nurses may leads to negative many negative consequences in them (Hsiao et al., 2011).

There are many studies which explore conscience of nurses in western context. For example the findings of studies in Finland (Saarnio et al., 2012) and Sweden (Tuvesson et al., 2012; Ericson-Lidman and Strandberg, 2013) showed that nurses experiences high levels of stress of consciences. Time pressure and high work load were the main causes that caused stress of conscience (Juthberg and Sundin, 2010; Ahlin et al., 2013). To the best of our knowledge, there are inadequate quantitative studies that examined level of conscience among Iranian nurses. However, the findings of some qualitative studies showed that conscience is an important factor in delivering nursing care among Iranian nurses (Vanaki and Memarian, 2009; Vaismoradi et al., 2011). On the other hand, in wide review of relevant literature we found no studies investigated the level of conscience among nurses who working in oncology wards. It should be noted that exploring the level of conscience and its related factors among oncology nurses could provide valuable information regarding the quality of ethic-based nursing care available for cancer patients. So, the purpose of this study was to examine the level of conscience and its related factors among Iranian oncology nurses.

\section{Materials and Methods}

This study has a descriptive-correlational design that was conducted in 3 hospitals in Tabriz, Iran. Tabriz is a capital city of East Azerbaijan Province, located in northwest of Iran. The study population includes 75 nurses who were working in these centers during the sampling period and met the following criteria: (a) having at least six months clinical experience; (b) qualified BSc or MSc degree in nursing; and (c) directly involved in oncology care. Due to the small study population, all of these nurses were included with census sampling method. Finally, 68 nurses accepted to be enrolled (response rate=91\%).

The questionnaire used in this study composed of two parts. First part was demographic checklist that investigates some socio-demographic and work-related characteristics of participants. The second part included the Perceptions of Conscience questionnaire (Dahlqvist et al., 2009). This scale has 15 items on a six-point Likert scale ranging from 1 (no, strongly disagree) to 6 (yes, strongly agree). The total score was ranging from 15 to 90 points (the higher scores indicating higher level of conscience).

For using the questionnaire, the instrument was translated into Persian and then back translated. Then, face and content validity of instrument were assessed and verified by the expert panel constituted fifteen faculty members affiliated to Tabriz university of Medical Sciences, Tabriz/Iran. The final version of the questionnaire was tested for reliability in a pilot study involving 20 nurses. Cronbach-Alpha coefficient value for Perceptions of Conscience questionnaire was 0.79 .

Before the data collection, the study was approved by Regional Ethics Committee of Tabriz University of Medical Sciences. Next, one of researchers was referred to hospitals in different shifts and identified the nurses who meet the inclusion criteria. Nurses who met criteria for the study were identified and all eligible samples were informed and invited to participate. All nurses who participated in the study gave informed consent according to ethic committee guideline. Willing nurses were asked to fill the questionnaire in their convenience time. Data collection lasted from September 2014 to February 2015.

Data were analyzed using SPSS statistical software (version 15). Descriptive statistics such as the frequency, percentage, mean and standard deviation were used to describe the demographic/professional-related data and level of conscience. Relationships between nurses' characteristics with levels of conscience were assessed by inferential statistics including independent t-tests, ANOVA and Pearson's correlation as appropriate.

\section{Results}

Analysis of some demographic characteristics of participants showed that most of them were male $(925 \%)$, married (794\%), and had rotation shifts (779\%). The mean of nurses' ages (years) and work experience (months) were $33.89(7.96)$ and 104.57 (18.25), respectively.

Table 1 shows the nurses' responses to all the items of Perceptions of Conscience questionnaire. As shown in this table the items "Our conscience warns us against hurting ourselves" and "God speaks to us through our conscience" had the highest and lowest scores, respectively. The study also showed that the mean of nurses' total score of the questionnaire was 72 which show high perceptions of

Table 1. Responses of nurses to all Items of Perceptions of Conscience Questionnaire

\begin{tabular}{|c|c|c|}
\hline Items & Mean & SD \\
\hline The voice of conscience must be interpreted & 5.26 & 0.8 \\
\hline \multicolumn{3}{|c|}{ You need inner peace to be able to hear the voice of conscience } \\
\hline & 5.33 & 0.76 \\
\hline We cannot avoid the voice of conscience & 5.31 & 0.85 \\
\hline \multicolumn{3}{|l|}{ Our conscience warns us against hurting ourselves } \\
\hline & 5.48 & 0.57 \\
\hline Our conscience warns us against hurting others & 4.86 & 1.21 \\
\hline \multicolumn{3}{|c|}{ We should follow our conscience, no matter what other people } \\
\hline think & 5.08 & 0.91 \\
\hline \multicolumn{3}{|c|}{ At my workplace I can express what my conscience tells me } \\
\hline & 5.16 & 0.89 \\
\hline I follow my conscience in my work & 3.75 & 1.63 \\
\hline Our conscience can give us the wrong signals & 4.95 & 1.17 \\
\hline Our conscience weakens if we do not listen to it & 5.07 & 0.8 \\
\hline \multicolumn{3}{|c|}{ I have to deaden my conscience in order to keep working in } \\
\hline health care & 5.2 & 0.8 \\
\hline My conscience is far too strict & 4.03 & 1.73 \\
\hline Our conscience expresses our social values & 4.57 & 1.38 \\
\hline God speaks to us through our conscience & 3.69 & 1.59 \\
\hline \multicolumn{3}{|c|}{ When I follow my conscience I develop as a human being } \\
\hline & 4.94 & 1.04 \\
\hline Total score & 72.74 & 7.48 \\
\hline
\end{tabular}

$\mathrm{SD}=$ standard deviation 
Table 2. Responses of Nurses to Sub-scales of Perceptions of Conscience Questionnaire

\begin{tabular}{lcccc}
\hline Sub-scales & Mean & SD & $\begin{array}{c}\text { Mean (based } \\
\text { on 100) }\end{array}$ & $\begin{array}{c}\text { SD (based } \\
\text { on 100) }\end{array}$ \\
\hline Authority & 20.56 & 3.09 & 86.62 & 12.92 \\
Warning signal & 14.32 & 2.64 & 79.65 & 14.75 \\
Demanding sensitivity & 13.52 & 2.66 & 76.21 & 14.21 \\
Asset & 10.23 & 1.51 & 85.22 & 12.71 \\
Burden & 8.77 & 1.65 & 71.92 & 14.65 \\
Depending on culture & 9.12 & 1.44 & 75.94 & 14.33 \\
\hline
\end{tabular}

$\mathrm{SD}=$ standard deviation

Table 3. Relationship of Some Demographic and Profession Related Characteristics of Participants with Total Score of Conscience

\begin{tabular}{lllll}
\hline Variables & Sub-groups & Mean & SD & $\mathrm{p}$ \\
\hline Sex of nurse & Male & 75.64 & 3.77 & 0.39 \\
& Female & 72.71 & 7.56 & \\
Marital status & Single & 72.46 & 7.75 & 0.87 \\
& Married & 72.81 & 7.48 & \\
Working schedule & Rotation shift & 72.02 & 7.82 & 0.16 \\
& Fixed shift & 75.1 & 5.74 & \\
Sex of patients & Female & 73.95 & 5 & 0.71 \\
& Male & 73 & 11.74 & \\
\multirow{2}{*}{ Age } & Both of them & 72.26 & 8.1 & \\
Job experience & r=0.17 & & $\mathrm{p}=0.18$ & \\
\hline
\end{tabular}

conscience among nurses.

Table 2 shows the mean of nurses' scores in the six sub-scales of Perceptions of Conscience Questionnaire. As seen in this table in the Authority and Asset sub-scales nurses acquired higher scores. Also the mean of nurses' scores in Burden and Depending on culture sub-scales were the least.

Table 3 shows the relationship of some demographic and profession related characteristics of participants with total score of conscience. As shown in this table there was no statistical differences between these characteristics and the total score of conscience in participants.

\section{Discussion}

The aim of this study was to examine the level of conscience and its related factors among Iranian nurses. According to extensive literature review, this is one of the first studies which examined the level of conscience and its related factors among Iranian nurses. The study findings showed nurses reported high levels of conscience. Accordingly, the findings of studies conducted in developed countries showed high levels of stress of conscience among nurses. For example, the findings of a study in Sweden revealed that nurses in psychiatry ward experienced high levels of stress of conscience than nurses working in other wards (Tuvesson et al., 2012). Another study also reported high levels of stress of conscience among Sweden nurses. They also showed that nurses with higher levels of stress of conscience or those who perceived conscience as "Burden" experienced higher job burnout (Gustafsson et al., 2010). Also, other study also reported high levels of conscience tension among nurses in Finland mainly due to high workload and lack of time (Saarnio et al., 2012).

It should be noted that in an extensive review of literature we could not find a study which examined levels of conscience among Iranian nurses with quantitative approach. However, there are some clues in qualitative studies. According to a study Iranian nurses in intensive care wards believed that conscience is an important factor in providing care in such units (Shorideh et al., 2012). The findings of another study also showed that conscience is one the important modulators of Iranian nurses burnout (Rafii et al., 2004). Also, other Iranian study also found that conscience is an important factor for managing pain in patients by Iranian nurses (Hashemi et al., 2012). Conscience is an important concept for nurses. One of nurses' codes of ethic in Iran is conscience (Ravari et al., 2013; Zahedi et al., 2013). Conscience is also an important factor for professional commitment (Mahmoodishan et al., 2010). However, the negative impacts of conscience on nurses has been mentioned is some literature. For example, the findings of study in Iran showed that conscience is one of the main sources of stress among Iranian nurses due to confronting with ethical dilemmas and lack of time and resources (Najimi et al., 2012). So, the findings of this study indicate a high level of conscience in Iranian nurses and the consequences of such conscience need to be investigated further.

Dissimilar to our findings, some previous studies showed that female nurses had more job conscience than their male counterparts (Juthberg et al., 2007; Ahlin et al., 2013). However, few studies are available in regard to related factors of levels of conscience among Iranian nurses. So, because of low sample size in present study exploring all the factors affecting nurses' level of conscience requires further examination.

The study findings have implication for promoting nursing care. According to our findings oncology nurses had high levels of conscience. They also had positive perception regarding conscience-based caring in the workplace which must be considered in designing and implementing care plans. This can be a strong point for better planning of nursing care for cancer patients.

Despite the strength of this study, it also has some limitations. First, in this study nurse levels of conscience were examined based on their self-report which can affect the strength of findings. Also, the study was done in a province in north-western of Iran. So, it is not represent variation of all the country population. Also, the sample size of this study is low. As a result, a final conclusion about the relationship between conscience and some characteristics of nurses is difficult. In relation to future research, replicating such studies in other Iranian cultures as well as, Middle East countries is required. Finally, understanding all factors that affect nurses' perception of conscience requires further studies.

\section{Acknowledgements}

This is a report of a database from Master of Sciences thesis in nursing approved by Tabriz University of Medical Sciences. The authors wish to acknowledge all of the 
Behrang Gorbanzadeh et al

patients, whose contribution enabled the production of this article.

\section{References}

Abbasnezhad M, Rahmani A, Ghahramanian A, et al (2015). Cancer care burden among primary family caregivers of iranian hematologic cancer patients. Asian Pac J Cancer Prev, 16, 5499-505.

Abdollahzadeh F, Moradi N, Pakpour V, et al (2014). Un-met supportive care needs of Iranian breast cancer patients. Asian Pac J Cancer Prev, 15, 3933-8.

Ahlin J, Ericson-Lidman E, Eriksson S, et al (2013). Longitudinal relationships between stress of conscience and concepts of importance. Nurs Ethics, 20, 927-42.

Atashzadeh Shorideh F, Ashktorab T, Yaghmaei F (2012). Iranian intensive care unit nurses' moral distress: a content analysis . Nurs Ethics, 19, 464-78.

Avestan Z, Rahmani A, Heshmati-Nabavi F, et al (2015). Perceptions of iranian cancer patients regarding respecting their dignity in hospital settings. Asian Pac J Cancer Prev, 16, 5453-8.

Dahlqvist V, Soderberg A, Norberg A (2009). Facing inadequacy and being good enough: psychiatric care providers' narratives about experiencing and coping with troubled conscience. $J$ Psychiatr Ment Health Nurs, 16, 242-7.

Ericson-Lidman E, Strandberg G (2013). Dealing with troubled conscience in municipal care of older people. Nurs Ethics, 20, 300-11.

Fathollahzade A, Rahmani A, Dadashzadeh A, et al (2015). Financial distress and its predicting factors among iranian cancer patients. Asian Pac J Cancer Prev, 16, 1621-5.

Glasberg AL, Eriksson S, Dahlqvist V, et al (2006). Development and initial validation of the Stress of Conscience Questionnaire. Nurs Ethics, 13, 633-48.

Gustafsson G, Eriksson S, Strandberg G, et al (2010). Burnout and perceptions of conscience among health care personnel: a pilot study. Nurs Ethics, 17, 23-38.

Hashemi F, Nasrabadi AN, Asghari F (2012). Factors associated with reporting nursing errors in Iran: a qualitative study. BMC Nurs, 11, 20.

Hsiao SM, Gau ML, Ingleton C, et al (2011). An exploration of spiritual needs of Taiwanese patients with advanced cancer during the therapeutic processes. J Clin Nurs, 20, 950-9.

Jensen A, Lidell E (2009). The influence of conscience in nursing. Nurs Ethics, 16, 31-42.

Juthberg C, Eriksson S, Norberg A, et al (2007). Perceptions of conscience in relation to stress of conscience. Nurs Ethics, 14, 329-43.

Juthberg C, Eriksson S, Norberg A, et al (2008). Stress of conscience and perceptions of conscience in relation to burnout among care-providers in older people. J Clin Nurs, 17, 1897-906.

Juthberg C, Sundin K (2010). Registered nurses' and nurse assistants' lived experience of troubled conscience in their work in elderly care--a phenomenological hermeneutic study. Int J Nurs Stud, 47, 20-9.

Mahmoodishan G, Alhani F, Ahmadi F, et al (2010). Iranian nurses' perception of spirituality and spiritual care: a qualitative content analysis study. J Med Ethics Hist Med, 3, 6 .

Najimi A, Goudarzi AM, Sharifirad G (2012). Causes of job stress in nurses: A cross-sectional study. Iran J Nurs Midwifery Res, 17, 301-5.

Rafii F, Oskouie F, Nikravesh M (2004). Factors involved in nurses' responses to burnout: a grounded theory study. BMC Nurs, 3, 6.
Ravari A, Bazargan-Hejazi S, Ebadi A, et al (2013). Work values and job satisfaction: a qualitative study of Iranian nurses. Nurs Ethics, 20, 448-58.

Saarnio R, Sarvimaki A, Laukkala H, et al (2012). Stress of conscience among staff caring for older persons in Finland. Nurs Ethics, 19, 104-15.

Tuvesson H, Eklund M, Wann-Hansson C (2012). Stress of Conscience among psychiatric nursing staff in relation to environmental and individual factors. Nurs Ethics, 19, 208-19.

Vaismoradi M, Salsali M, Ahmadi F (2011). Perspectives of Iranian male nursing students regarding the role of nursing education in developing a professional identity: a content analysis study. Jpn J Nurs Sci, 8, 174-83.

Vanaki Z, Memarian R (2009). Professional ethics: beyond the clinical competency. J Prof Nurs, 25, 285-91.

Weaver K (2007). Ethical sensitivity: state of knowledge and needs for further research. Nurs Ethics, 14, 141-55.

Wengstrom Y, Ekedahl M (2006). The art of professional development and caring in cancer nursing. Nurs Health Sci, 8, 20-6.

Yeh CH, Lin CF, Tsai JL, et al (1999). Determinants of parental decisions on 'drop out' from cancer treatment for childhood cancer patients. J Adv Nurs, 30, 193-9.

Zahedi F, Sanjari M, Aala M, et al (2013). The code of ethics for nurses. Iran J Public Health, 42, 1-8. 